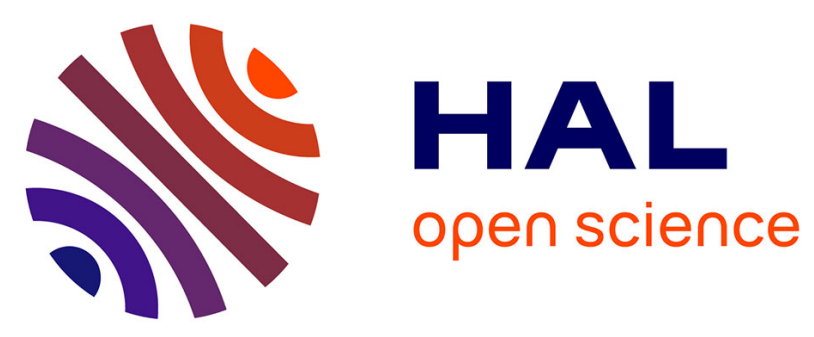

\title{
La programmation du développement régional. Des velléités à l'utopie réaliste: le cas de la Toscane
}

Giuliano Bianchi, Nicola Bellini, Emmanuel Négrier

\section{To cite this version:}

Giuliano Bianchi, Nicola Bellini, Emmanuel Négrier. La programmation du développement régional. Des velléités à l'utopie réaliste: le cas de la Toscane. Pôle Sud - Revue de science politique de l'Europe méridionale, 1998, Elections et politiques régionales., 8, pp.98-113. 10.3406/pole.1998.997. hal-02528250

\section{HAL Id: hal-02528250 \\ https://hal.science/hal-02528250}

Submitted on 24 Apr 2020

HAL is a multi-disciplinary open access archive for the deposit and dissemination of scientific research documents, whether they are published or not. The documents may come from teaching and research institutions in France or abroad, or from public or private research centers.
L'archive ouverte pluridisciplinaire $\mathbf{H A L}$, est destinée au dépôt et à la diffusion de documents scientifiques de niveau recherche, publiés ou non, émanant des établissements d'enseignement et de recherche français ou étrangers, des laboratoires publics ou privés.

\section{(ㅇ)(1) $\$$}

Distributed under a Creative Commons Attribution - NonCommercial - NoDerivatives $\mid 4.0$ 
La programmation du développement régional. Des velléités à I'utopie réaliste : le cas de la Toscane

Mr Giuliano Bianchi, Mr Nicola Bellini, Mr Emmanuel Négrier

\section{Citer ce document / Cite this document :}

Bianchi Giuliano, Bellini Nicola, Négrier Emmanuel. La programmation du développement régional. Des velléités à l'utopie réaliste : le cas de la Toscane. In: Pôle Sud, n², 1998. Elections et politiques régionales. pp. 98-113;

doi : https://doi.org/10.3406/pole.1998.997

https://www.persee.fr/doc/pole_1262-1676_1998_num_8_1_997

Fichier pdf généré le 23/04/2018 


\title{
La programmation du développement régional Des velléités à l'utopie réaliste : le cas de la Toscane
}

\author{
par Giuliano Bianchi, Université de Sienne \\ et Nicola Bellini, Université de Pise \\ (Traduit de l'italien par $E$. Négrier) \\ Pôle Sud $N^{\circ} 8-$ mai $1998-p .98$ à 113.
}

\begin{abstract}
Cet article reconstitue synthétiquement le parcours culturel et politique de l'activité de programmation de la Région Toscane dans sa formulation et son gouvernement du développement économique territorial. Ce premier paragraphe retrace les étapes essentielles de l'évolution de la programmation régionale en Italie et en Toscane. Le second paragraphe présente les thèmes des plus récentes réflexions sur le développement toscan et leurs implications politiques majeures. Le dernier paragraphe propose une analyse des principales innovations introduites par le Programme Régional de Développement (PRS) 1998-2000 de la Toscane, approuvé en décembre 1997.
\end{abstract}

La trajectoire de la programmation régionale en Toscane et en Italie

Cet article reconstitue synthétiquement le parcours culturel et politique de l'activité de programmation de la région Toscane dans sa formulation et son gouvernement du développement économique territorial. Ce premier paragraphe retrace les étapes essentielles de l'évolution de la programmation régionale en Italie et en Toscane. Le second paragraphe présente les thèmes des plus récentes réflexions sur le développement toscan et leurs implications politiques majeures. Le dernier paragraphe propose une analyse des principales innovations introduites par le Programme Régional de Développement (PRS) 1998-2000 de la Toscane, approuvé en décembre 1997.

Avec les limites que nous signalerons, la programmation a été un thème majeur d'affirmation du nouvel échelon de gouver- nement régional en Italie : même lorsque ce n'était qu'un exercice rhétorique - et de facto un aveu d'impuissance - la programmation a servi à construire l'identité de la nouvelle institution, à en former les dirigeants politiques et administratifs et à les rendre conscients des limites et des potentialités propres d'un niveau meso de gouvernement.

L'expérience de la Toscane, tout en reproduisant en maints aspects des caractéristiques historiques et institutionnelles typiquement italiennes, représente selon nous un cas intéressant pour ses propriétés particulières : une structure économique largement caractérisée par le phénomène des districts industriels (Bellini, 1996); un processus complexe de dévolution de compétences administratives et de gestion de la région aux gouvernements locaux pour spécifier ses propres fonctions d'orientation politique, programmatique et de contrôle; 


\section{La programmation du développement régional}

un débat vivace entre options industrialiste et post-industrialiste.

La programmation régionale en Italie a dû avant tout affronter l'asymétrie entre pouvoirs et ressources effectivement disponibles et l'ambition de donner au nouveau pouvoir régional la mission d'orienter le développement socio-économique. Si on retrouve - même s'ils sont peu homogènes les ingrédients techniques typiques de la planification (analyse des structures régionales, définition et quantification des priorités, procédure d'évaluation et de contrôle) dans la méthodologie de programmation, les possibilités d'action concrète restent limitées. Les politiques macro-économiques, notamment celles qui ont eu une fonction centrale dans le soutien au développement (on pense aux politiques de valorisation et de soutien à l'exportation), demeurent interdites à la région. Mais le noyau dur des politiques industrielles, y compris à destination des PMI, est soustrait à l'intervention régionale.

Cependant, la programmation régionale est née avant même la constitution effective des régions, avec la première élection des conseillers régionaux le 7 juin 1970, lorsque celles-ci n'étaient, à l'exception des régions à statut spécial, qu'une mention virtuelle de la Constitution, une liste de noms sans identité politique et parfois historique, bref un pur phénomène géographique (Bianchi, 1982).

Les années 1950 et 1960 sont, de ce point de vue, des années très importantes qui ont contribué à déterminer les différenciations entre styles régionaux de gouvernement et de programmation. Ce sont les années de la redécouverte des déséquilibres territoriaux comme phénomène plus complexe et contrasté que réduit à la "question méridionale". Les activités de programmation sont mises en œuvre par des initiatives spécifiques des administrations provinciales et des chambres de commerce, "préfigurant" de fait la Région-institution.

Ce sont par ailleurs les années du développement à l'échelle nationale - lancées puis défaillantes en termes de résultats concrets - de la programmation, objet d'une réflexion souvent originale, et des propositions de quelques grandes personnalités catholiques (Vanoni, Saraceno) et laïques (La Malfa) (Garabba, 1977; Ruffolo, 1980). Au niveau régional, cette attention se traduit en "exercices" de programmation destinés à définir des "platesformes régionales" de développement. L'élite de l'intelligence économique italienne (Lombardini, Gasperini, Fuà, Sylos, Labini) y contribue de manière déterminante, et dans certains cas par une extraordinaire mobilisation de compétences et de technologies '. Ceci se produisit ainsi en Toscane où, sous l'impulsion pionnière de Giacomo Becattini ${ }^{2}$, se développa une attention aux caractéristiques d'un développement original fondé sur des agglomérations territoriales de petites entreprises spécialisées dans des productions traditionnelles (qui seront par la suite appelées, grâce encore à Becattini, "districts industriels" ${ }^{3}$ ). Ce travail de "programmation sans institution", cent ans après l'unification italienne, en montrait l'absence d'unification économique (Saraceno, 1961) par une reconstitution de l'identité perdue des régions et de leurs niveaux différents de développement. 


\section{Pôle Sud $N^{\circ} 8$}

Les groupes dirigeants qui, à compter de 1970 , se retrouveront dans les institutions régionales, se mettent en avant dans cette expérience.

Le panorama de la programmation régionale italienne devient rapidement contrasté. L'expérimentation n'a pas lieu partout. A la fin des années 60 , le gouvernement central lui-même qui institue par la loi des "comités régionaux pour la programmation économique", qui ont pour tâche d'élaborer des plans, y compris dans les territoires où aucune initiative de base ne s'était manifestée.

On notera que la programmation ne décolle pas dans les régions dotées d'un statut spécial dès la fin des années 1940. Les bases légales ne manquent pas. En Sicile, le statut, approuvé avant la Constitution, parlait explicitement de "planification". Le statut de la Sardaigne spécifiait qu'il s'agissait là de "plan de renaissance". Peu advint dans les faits. Dans quelques régions à statut spécial, les situations spécifiques restaient étrangères à l'approche programmatique, de fait "intraitable" dans ces institutions : il suffit de penser à la question ethnique dans le Trentin-Haut-Adige. Encore une fois, il n'y a pas de relation entre programmation et pouvoirs : ce n'est pas l'ampleur de ceuxci qui induit l'effort de programmation. Il est significatif que les plans élaborés ici ne sont pas le fruit de compétences locales mais d'apports externes. Le plan n'est pas tant l'expression d'un projet politique qu'une base de revendication à l'égard du gouvernement central; en ce sens, il conduit plus à une déresponsabilisation diffuse qu'à l'émergence d'une culture de gouvernement.
Avec la constitution des régions, l'activité de programmation est évidemment relancée. Au cours des années 1970, apparaît une centaine d'exercices, souvent marqués par une approche d'abord territoriale; un choix incontestablement réaliste dans la mesure où il se réfere à la compétence la plus significative attribuée aux régions : l'approbation des plans d'urbanisme municipaux. Dans d'autres cas, on s'exerce en revanche à définir des hypothèses de développement économique, pour la réalisation desquelles les régions ne possèdent pas de réels pouvoirs et sont donc destinées à demeurer au stade des aspirations.

Les années 1970 sont essentiellement, pour la programmation régionale, des années d'illusion. Les plans suivent avant tout une approche globale pour maîtriser les processus économiques, sociaux et territoriaux du développement. C'est une programmation impotente qui s'assigne des objectifs ne pouvant être atteints que très marginalement.

Toutefois, l'apparition des nouvelles autorités régionales ne reste pas sans effet. Face à des politiques concrètes (la possibilité d'interagir avec les entreprises par le soutien aux investissements ou par la formation professionnelle; la naissance des premières agences régionales de développement, etc.), les plans régionaux, malgré leur manque d'efficacité : représentent une référence pour l'activité législative et administrative, accroissant la possibilité de contrôle critique des assemblées de conseillers sur les exécutifs; offrent donc aux oppositions la possibilité d'identifier et de dénoncer les éventuelles contradictions entre déclarations d'intentions et pratiques 


\section{La programmation du développement régional}

quotidiennes; favorisent la relation dialectique avec la région pour les institutions locales et les forces sociales; fournissent la base la plus correcte aux négociations entre la région et le gouvernement central.

Le Dpr 616 de 1977, acte qui signe le premier transfert massif de compétences entre l'État et les régions, établit que le bilan régional doit faire partie d'un bilan triennal, qui doit lui-même trouver sa référence dans un "programme régional de développement" (PRS) correspondant. Jusqu'à aujourd'hui, c'est la première et la seule référence légale nationale en cette matière : le PRS conditionne l'approbation du budget régional par l'État. Cette norme, qui consacre la nécessité du PRS, en diminue en même temps la portée politique. De fait, il l'identifie comme une annexe budgétaire, à peine plus qu'un exercice bureaucratique, où manque une réelle volonté politique originale. Rien n'est dit non plus sur les contenus des PRS, ce qui est d'autant plus gênant que, dans le même temps, fleurissent dans d'autres pays européens ${ }^{4}$ de nouvelles théories, méthodologies et techniques de la programmation régionale et locale.

Les années 1980 sont celles de la désillusion et du redimensionnement des ambitions de la programmation. L'écho des fondements culturels et théoriques des origines et les "livres des songes" semblent à jamais enfouis au nom du réalisme. On renonce à élaborer des stratégies de développement et le PRS devient la liste organisée des projets et de leurs illustrations à des fins budgétaires.

L'approche par projet n'est certes pas sans mérite, en portant l'attention sur les aspects opérationnels du gouvernement. On ren- verse les relations entre théorie et pratique et, si les plans sont moins brillants en termes de documents, ils enrichissent les politiques avec une gamme nouvelle d'actions concrètes et d'instruments. On anticipe (spécialement en Toscane et en Emilie-Romagne) le modèle de sélection des projets par appel d'offre, qui deviendra ensuite la norme européenne. Aux initiatives de promotion s'associent, en particulier en Lombardie et en Emilie-Romagne, les premières interventions en matière de développement technologique. Les instruments typiques des politiques régionales (sociétés financières, agences de développement) se consolident, parfois au prix de choix différents : en Toscane, l'agence de promotion de l'artisanat est ainsi fermée. Les expériences des centres de services aux entreprises voient le jour, qui, sans être une invention italienne, y ont leurs lettres de noblesse théorique, et sont reliées au modèle des systèmes territoriaux des PME.

À la fin des années 1980 s'ouvre, pour la programmation régionale italienne, une phase d'intenses réflexions. Il y a à tirer les leçons critiques des expériences menées jusque-là, où continuent de faire systématiquement défaut les éléments de définition de la politique de planification par rapport à d'autres actions économiques : la quantification des objectifs, l'évaluation ex-ante des alternatives, le management des effets ex-post.

Dans le même temps, dans la grande majorité des régions, les difficultés que le PRS et les plans sectoriels posent à la définition des budgets sont faibles ou seulement partiellement significatifs. La satisfaction de l'appareil analytique, produit par des consultants externes ou par des instituts régionaux de recherche, provient de l'adoption de 


\section{Pôle Sud $N^{\circ} 8$}

méthodes formalisées et surtout de l'analyse coûts-bénéfices. Toutefois, ce n'est souvent qu'une rigueur instrumentale pour garantir une adéquation formelle aux obligations imposées par les procédures nationales ou européennes. La Toscane, sur ce terrain, se distingue par un effort à construire, entre 1979 et 1984 , un "système de modèles pour l'analyse de la région Toscane" (SMART), réalisé par l'institut IRPET (Bianchi, 1983; Cavalieri, Martellato, Snickars, 1984). Le "cœur" du système est un modèle input/output birégional (Toscane et reste de l'Italies).

Le véritable élément de nouveauté est toutefois la fréquentation de plus en plus poussée des approches européennes ${ }^{6}$. L'expérience des PIM (Programmes Intégrés Méditerranéens) est décisive en ce sens parce que, s'ils donnent lieu à la première expérience de programmation régionale à grande échelle, ils obligent, au niveau national, à se familiariser avec le concept de programmation intégrée (et non plus sectorielle), d'évaluation et de management, un vocabulaire jusqu'alors absent du lexique politico-administratif. L'importance des PIM dépasse ainsi les résultats concrets obtenus sur un plan purement technique : l'effet d'innovation se traduit au contraire sur le plan politique (Bianchi, 1990, 1992). Avec les PIM s'expérimente avant tout dans 29 régions européennes, pas particulièrement avancées, une négociation trilatérale, Commission Européenne/Gouvernements nationaux/ régions, qui reconnaît finalement à ces dernières un statut égal aux autres niveaux de gouvernement. Deuxièmement, la programmation contractualisée, pose des contraintes qualitatives à l'action, impose des délais de rigueur, prévoit des sanctions en cas de carence. En troisième lieu, les PIM obligent les agents destinataires à cofinancer les interventions et donc à une cohérence de comportement au nom de l'additionnalité des ressources européennes : c'est la consécration de la méthode partenariale. En réalité, les PIM connaissent leurs meilleurs résultats là où les acteurs privés participent aux projets non en termes de consensus générique mais en termes d'emploi concret de ressources.

Les PIM ont ainsi constitué le laboratoire d'une méthode qui sera généralisée à l'échelle européenne avec la réforme des fonds structurels. Au niveau régional, l'effet le plus évident est celui de la formation, sur le style européen, d'une nouvelle génération de fonctionnaires publics. C'est ce style qui fournira les nouvelles références de la programmation régionale, même si elles pénètrent sans véritable prise de conscience de la part des institutions, mais par un processus quasi spontané de learning by doing.

Cet impact n'est pas le résultat d'une action véritable de la part de l'Union européenne. Respectant une règle de non-interférence, la Commission n'intervient pas sur l'amélioration des structures administratives : l'objectif de cohésion économique et sociale ne rejoint pas celui de cohésion institutionnelle pour réduire les écarts d'efficience des administrations publiques, notamment régionales et locales. Le creusement ultérieur des différences de performance des institutions italiennes ne saurait surprendre. Il y a en fait des régions qui montrent à la fois des capacites d'initiative et de gestion, organisant leur propre présence à Bruxelles et promouvant 


\section{La programmation du développement régional}

des alliances inter-régionales (c'est le cas de l'Émilie-Romagne et de la Lombardie). Un second groupe de régions, parmi lesquelles figurent la Toscane et les régions du Centre et du Nord-Est en général, expriment une bonne voire excellente capacité de gestion administrative de leurs interventions, mais ne semblent pas encore disposer d'une capacité de projet, toujours plus nécessaire, dans le scénario de renforcement de la compétition qui s'annonce dans les évolutions actuelles des politiques communautaires. $\AA$ plus forte raison, ce scénario apparaît problématique pour le troisième groupe de régions, qui comprend la quasi-totalité des régions méridionales : la faiblesse ou l'inexistence de projet se combine à des situations de pathologique inefficience administrative qui a conduit à d'embarrassants retards dans la mise en œuvre des programmes.

\section{Une nouvelle vision du développe- ment économique toscan : les implications pour la stratégie régionale}

Dans les dernières années, on a enregistré en Toscane un aggiornamento significatif et parfois radical des connaissances sur les tendances du développement, avec des implications importantes pour l'identification des grandes options stratégiques qui en découlaient ${ }^{7}$.

Sans aucun doute, la Toscane se présente aisément dans le groupe des régions européennes les plus évoluées au rendez-vous de l'an 2000, que ce soit sur le plan social qu'en termes de performances économiques. L'aspect que présente l'industrie toscane en cette fin de siècle est toutefois un clair-obscur d'ombre et de lumière qui a justifié des interprétations très divergentes, tendant à en accentuer les problèmes ou, inversement, à rassurer sur ses potentiels compétitifs. En réalité, il n'y a pas d'alternative à une lecture nuancée, même si plutôt positive dans l'ensemble.

Les données préoccupantes sont sans équivoque : à partir des années 1980 , la base productive a subi une contraction de type structurel. En 1970, le secteur industriel pesait $42,6 \%$ de l'économie régionale, en $1994,31 \%$, et une baisse ultérieure est prévue jusqu'à la fin de la décennie. Il est cependant aussi vrai qu’à partir de 1993, on enregistre une croissance de la productivité, qui a remis l'industrie toscane au niveau des taux de croissance des zones les plus dynamiques du pays.

La contraction répond naturellement à des tendances structurelles de longue période dans l'ensemble des économies développées qui voient le déplacement de la demande des biens aux services, soit en raison de l'évolution vers l'immatériel des activités productives, soit à cause de l'expansion d'un tertiaire plus ou moins innovant. Le futur proche de la Toscane risque cependant de n'être ni néo-industriel ni post-industriel.

D'un côté, la désindustrialisation a eu des effets négatifs sur le secteur des services innovants aux entreprises et donc sur l'opportunité d'évoluer vers des systèmes technologiques et organisationnels nouveaux (de l'entreprise en réseau à la lean production). D'un autre côté, la Toscane a accentué sa propre spécialisation dans les services à la personne, que d'aucuns considèrent comme la vocation de nouveaux modèles de développement, mais qui ont 


\section{Pôle Sud $N^{\circ} 8$}

des niveaux de productivité et de compétitivité encore trop bas. En termes de richesse, les services à la personne ne paraissent pas incarner une réelle alternative à la centralité de l'industrie, laquelle requiert une amélioration et un renforcement de ses contenus innovants.

Certains aspects de l'industrie toscane nécessitent évidemment une reconsidération critique. À la base de sa perte relative de vitesse vis-à-vis d'autres aires italiennes au cours des années 1980, on trouve clairement : l'épuisement des avantages originaux de flexibilité et d'élasticité (qu'on ne trouve plus exclusivement dans le système des PMI); la faible propension à investir, ajoutée à des formes poussées de rationalisation productive et entrepreneuriale; une pénurie préoccupante d'entreprises moyennes et de leaders.

On doit souligner combien le déplacement compétitif n'est pas le résultat automatique de recompositions sectorielles (ou bien de la baisse structurelle de secteurs traditionnellement locomotives régionales), même si on ne peut nier qu'aux difficultés des biens de consommation, dues à l'élévation des seuils critiques d'investissement en $\mathrm{R} \& \mathrm{D}$ et de la capacité de maîtrise des marchés, ont correspondu une performance bien meilleure dans les industries des biens intermédiaires. Ici (textile, cuirs et peaux, papier) ont compté des facteurs décisifs comme le contrôle technique des processus, la capacité d'interagir avec l'environnement, la densité des systèmes de district industriel, le contrôle des approvisionnements.

Au-delà de l'appartenance sectorielle, il existe en effet en Toscane un noyau d'entreprises dynamiques et innovatrices, qui a vécu dans ces années une croissance très soutenue, a introduit des innovations organisationnelles significatives (avec le recrutement de managers, des délégations de fonctions, l'intégration de nouveaux rôles), a fait évoluer les rapports entre fournisseurs, consolidé une maîtrise technologique spécialisée, innové dans son rapport au marché et a recherché (sans toujours les trouver) de nouvelles solutions de financement. Ces comportements vertueux indiquent clairement qu'il existe une excellence réelle, praticable et possible dans le contexte toscan : la condamnation au déclin industriel est loin d'être écrite. Dans le même temps, ces expériences sont quantitativement insuffisantes pour être le moteur d'une nouvelle phase de développement : elles sont trop peu nombreuses par rapport à l'ensemble des entreprises; elles sont trop petites pour avoir un effet d'entraînement sur le territoire.

Du point de vue conceptuel, en référence et en discontinuité vis-à-vis du passé, la principale conséquence politique de cette analyse tient dans l'impossibilité de proposer un modèle unique de référence, sur lequel faire converger les comportements des acteurs économiques. Il n'existe donc pas d'espace pour des stratégies de type dirigiste : la principale préoccupation doit être non pas celle de définir a priori une trajectoire de développement, mais de construire les conditions pour une variété d'options, non nécessairement alternatives, qui puissent exprimer dans le temps leurs propres potentialités.

Les stratégies "flexibles" présupposent un choix précis des variables structurelles sur lesquelles on entend agir. En particulier, 


\section{La programmation du développement régional}

elles doivent avoir les caractéristiques de manœuvrabilité, ou bien une sensibilité effective aux politiques publiques; de multiplicateur politique de l'intervention, dans le sens d'induire ou d'offrir une stimulation et des opportunités à des comportements cohérents et synergiques de la part des autres acteurs, en amplifiant ainsi l'impact des politiques et en donnant naissance à des processus de changement plus profonds et de plus long terme.

Les variables qui ont ces caractéristiques en Toscane aujourd'hui sont au nombre de trois : (i) la variété des modèles et des moteurs du développement et, en ce sens, le poids et la capacité d'entraînement du secteur manufacturier; (ii) le degré d'innovation du système; (iii) l'intégration internationale de l'économie toscane.

- La variété des modèles de développement. L'existence d'une pluralité de parcours représente une condition nécessaire pour garantir à l'économie toscane une réponse efficace à plusieurs scénarios possibles de concurrence, encore difficilement prévisibles face à la reconfiguration en cours de l'économie internationale. À côté d'activités qui offrent d'importantes perspectives de développement, le secteur manufacturier demeure incontournable comme secteur pilote. Au contraire, son évolution propre en accentue la fonction d'induction de l'innovation dans le système économique et dans la société. L'industrie demeure en outre l'espace où la compétition globale s'exprime avec l'impact le plus fort, notamment en termes d'orientation et d'expérimentation.

- Le degré d'innovation du système. Celuici ne se réduit pas à la dimension technolo- gique, mise en œuvre par le système des entreprises. Il se réalise dans la poursuite cohérente (et souvent avec des phases d'accélération et de discontinuité) des changements dans leur profil stratégique, notamment sur leur modalité de présence sur le marché, les processus d'internationalisation, les formes d'organisation des relations entre entreprises et le bénéfice tiré des situations dominantes en matière de technologies spécifiques. L'innovation de profil stratégique dépend donc souvent de changements quant à l'emploi des ressources (humaines, technologiques et financières).

- Lintégration internationale de l'économie toscane. Elle comporte la constitution d'un capital de relations et de positionnements sur les réseaux financiers, technologiques et entrepreneuriaux d'échelle globale. Par ailleurs, la capacité de participer à la dynamique concurrentielle internationale "impose" au système productif et à la région elle-même une adaptation aux standards de qualité et de compétitivité les plus élevées.

Pour quel changement agir sur de telles variables? Les analyses ont mis en évidence ces dernières années un "changement effectué", par l'effet des comportements innovatifs individuels et essentiellement autoengendrés par les entreprises. Ce changement a conduit à l'émergence d'un groupe d'entreprises d'excellence qui ont apporté la preuve que l'innovation est possible, mais aussi celle du rapport de cause à effet entre innovation (lato sensu) et performance positive.

Pourtant, un changement possible est avant tout celui qui peut survenir par la diffusion de ces processus individuels. Il est 


\section{Pôle Sud $N^{\circ} 8$}

donc celui qui permet d'étendre les bénéfices liés aux virtualités actuelles du système toscan; celui-ci ne laisse pourtant pas prévoir un paysage réellement nouveau du système productif : il porte au rendement maximum un moteur existant, sans toutefois en changer.

On doit alors parler de changement souhaitable, seul résultat d'une mutation plus profonde concernant les facteurs concurrentiels et les "règles" du modèle toscan de développement. Ce changement fait évoluer les conditions de fond du système : il déplace la question de l'excellence d'une dimension purement individuelle, ainsi élargie à l'échelle d'un système. Il rassemble enfin les parcours innovants individuels dans un nouveau contexte, plus favorable et solide.

Ce schéma conceptuel définit les deux enjeux vis-à-vis desquels la politique régionale a potentiellement un rôle à jouer : celui du passage d'un changement effectué à un changement possible, ou encore celui de la diffusion des comportements innovants; celui du passage du changement possible au changement souhaitable : ou bien celui de l'innovation dans les facteurs de compétitivité du système productif régional ( $c f$. schéma $\mathrm{n}^{\circ} 1$ ).

Dans le premier passage, les politiques ont pour objectif d'accompagner la diffusion des comportements privés innovants. "Accompagner" signifie optimiser l'ensemble des actions qui constituent l'infrastructure de soutien, faite de règles, d'acteurs et de ressources, généralement disponibles dans les économies de marché industrialisées.

Face à cet enjeu, la politique industrielle agit selon des logiques de "conformité au marché" (market conforming). Il s'agit de politiques typiquement non dirigistes (ou alors gérées correctement) qui ne doivent pas distordre les mécanismes de marché et ne doivent pas tendre à dévier les orientations du développement, mais se limitent à renforcer la disponibilité et l'accessibilité des facteurs adaptés, et à atténuer les coûts et accélérer les délais d'ajustement.

Les politiques "conformes au marché" ont toutefois un rôle actif de soutien au changement et pas seulement d'élimination

\section{Changement et politique}

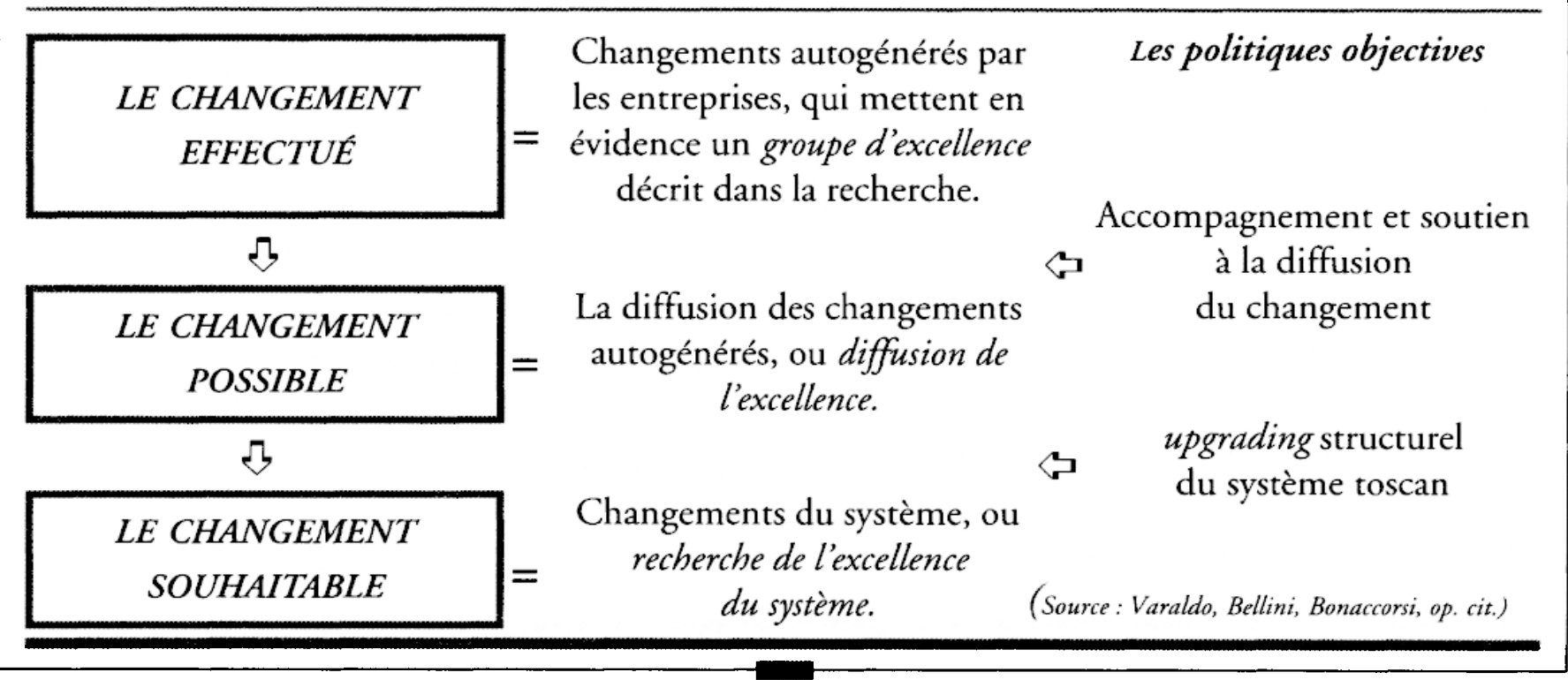




\section{La programmation du développement régional}

des obstacles bureaucratiques. Le soutien qu'ils apportent aux comportements entrepreneuriaux doit être en même temps :

- intelligent : pour une adaptabilité rapide face à la mutation des circonstances et des besoins, sur la base d'une capacité assez sophistiquée de lecture des tendances productives;

- systématique : pour s'entourer d'une large gamme d'instruments de réglementation, de stimulations infrastructurelles et de service, sans céder à la tentation (déjà vue par le passé) de tout faire reposer sur un seul instrument;

- constant : pour garantir des horizons stables et de long terme;

- fiable : pour garantir aux entreprises une bonne qualité de réponse des instruments politiques.

Les politiques de changement souhaitable se fixent explicitement l'objectif d'un saut qualitatif du système toscan. Elles représentent pour ce dernier de véritables investissements structurels, qui doivent être examinés et évalués. Les caractéristiques nécessaires à de telles politiques sont alors les suivantes :

- la capacité de donner un signe clair de discontinuité positive vis-à-vis du présent des atouts actuels de la structure productive et, lato sensu, de l'économie de la région;

- l'orientation vers les résultats (plutôt qu'à la fixation de normes, de procédures);

- l'acceptation d'objectifs de moyen et long terme (et donc celle d'une possible absence de rendement à court terme);

- l'acceptation d'une mesure raisonnable et pertinente du "risque" $d$ 'insuccès et d'évaluation imprécise (effets secondaires, indirects, de long terme);
- l'attribution de la priorité à des actions ponctuelles, praticables, mais dotées également d'un "multiplicateur politique", afin de réorienter et d'ordonner les comportements d'une pluralité d'acteurs;

- une "mentalité de projet" flexible et ouverte à des ajustements en cours ;

- une gestion fondée sur l'ingénierie d'un réseau de politique adapté, une véritable growth coalition qui joue un rôle de catalyseur en utilisant les ressources humaines, techniques, matérielles et politiques adéquates, et en renonçant sur une période suffisante à la possibilité de retour en arrière et à des conduites opportunistes.

Le schéma $\mathrm{n}^{\circ} 2$ articule les rapports entre les variables identifiées et ces "prescriptions" de politique, et spécifie les zones propres d'intervention les plus importantes.

\section{Le nouveau Programme Régional de Développement}

Le programme de développement (1998-2000) de la région Toscane, déjà en cours de mise en œuvre, représente la synthèse du parcours présenté ici et des révisions conceptuelles intervenues lors du débat régional. On met l'accent ici sur les innovations principales qui le caractérisent et qui sont aussi les aspects les plus problématiques pour la politique régionale du futur proche.

- la nouvelle diagnose du développement. Elle est principalement contenue dans le profil stratégique. On n'y traitait donc pas seulement d'une acquisition de type culturel, mais de la tentative de dépasser un déphasage historique entre les temps du développement et ceux de la politique. 


\section{Pôle Sud $N^{\circ} 8$}

\section{Stratégie, objectifs, politiques}

\begin{tabular}{|c|c|c|}
\hline $\begin{array}{c}\text { VARIABLES } \\
\text { STRUCTURELLES }\end{array}$ & $\begin{array}{r}\text { OBJECTIFS } \\
\text { POUR LE CHANGEMENT POSSIBLE }\end{array}$ & $\begin{array}{l}\text { ET POLITIQUES } \\
\text { POUR LE CHANGEMENT SOUHAITABLE }\end{array}$ \\
\hline $\begin{array}{l}\text { La variété des modèles et des } \\
\text { "moteurs" du développement et le } \\
\text { poids et la capacité d'entrầne- } \\
\text { ment du secteur manufacturier }\end{array}$ & $\begin{array}{l}\text { Un soutien "ouvert" à la crois- } \\
\text { sance des entreprises, non } \\
\text { contraignant, qui favorise la dif- } \\
\text { férenciation innovante des for- } \\
\text { mules entrepreneuriales et des } \\
\text { modèles d'organisation. }\end{array}$ & $\begin{array}{l}\text { L'augmentation de la différenciation } \\
\text { avec: } \\
\text { - le soutien dirigé vers les comporte- } \\
\text { ments innovants } \\
\text { - le soutien à la naissance de nou- } \\
\text { veaux acteurs privés, notamment } \\
\text { dans l'innovation (ex. spin-off) } \\
\text { - l'attraction d'entreprises exogènes }\end{array}$ \\
\hline $\begin{array}{l}\text { Le degré d'innovation } \\
\text { du système }\end{array}$ & $\begin{array}{l}\text { La diffusion des comportements } \\
\text { "vertueux". } \\
\text { Un "système régional de l'inno- } \\
\text { vazione" plus articulé et efficace } \\
\text { (rapport Université-entreprises, } \\
\text { services, financements inno- } \\
\text { vants). }\end{array}$ & $\begin{array}{l}\text { Le développement des systèmes } \\
\text { locaux. } \\
\text { Le développement du high tech, en } \\
\text { fonction (et attraction) d'interven- } \\
\text { tions de niveau national. } \\
\text { L'intégration du high tech dans le } \\
\text { système toscan (nouvelles modalités } \\
\text { du transfert de technologie; networ- } \\
\text { king pour l'innovation). }\end{array}$ \\
\hline $\begin{array}{l}\text { L'intégration internationale de } \\
\text { l'économie toscane }\end{array}$ & $\begin{array}{l}\text { Un soutien qualifié et dédié aux } \\
\text { processus d'internationalisation. }\end{array}$ & $\begin{array}{l}\text { L'homologation aux standards inter- } \\
\text { nationaux d'excellence territoriale, } \\
\text { notamment par le biais d'un pro- } \\
\text { gramme de marketing territorial. }\end{array}$ \\
\hline
\end{tabular}

C'est, en dernière analyse, la limite la plus grave que les politiques régionales ont jusqu'alors rencontrée. Si l'on considère, par exemple, les années 1950 à 1975, dominées par le succès du modèle de développement tiré par les systèmes territoriaux de petites entreprises spécialisées (Becattini, 1975), c'est seulement à la fin de cette période que l'on acquiert (avec un retard causé, entre autres facteurs, par l'idéologie de la gauche toscane) la conscience d'un tel modèle et qu'il se traduit en politiques, comme, par exemple, les services aux entreprises. De telles politiques ont au contraire fini par agir pendant la phase ultérieure, que l'on peut définir comme celle de la "maturité précoce" ${ }^{8}$ de l'appareil productif (19751990), dans un rôle passif de "résistance" à la trop rapide conversion vers le postindustriel, qui revenait pour beaucoup à de la désindustrialisation pure et simple. L'ambition du PRS est justement celle d'éviter le risque de définir des politiques pour une Toscane qui n'est plus et d'apprendre à raisonner et à agir en temps voulu sur les tendances effectivement en cours.

- Le rôle des processus innovants. La centralité des dynamiques innovantes est reconnue par le PRS comme le référentiel essentiel pour les politiques de développement. Des processus certes non absents de l'économie toscane. Les nouveaux savoirs 


\section{La programmation du développement régional}

sur le développement régional montrent l'importance des changements survenus, et le fait que l'accompagnement du "changement possible" (le soutien des tendances en cours) n'est pas particulièrement ardu; mais c'est l'innovation de système, parce qu'elle présuppose un effort sérieux de cohérence entre tous les acteurs (publics et privés), qui requiert un maximum de convergence des choix, des ressources et des attitudes.

Le défi le plus délicat réside clairement dans les politiques du "changement souhaitable". On pense à la complexité de l'innovation institutionnelle qui comporte des mutations de pouvoirs et de responsabilités (entre institutions et entre public et privé), politiquement et techniquement complexes. On pense à l'innovation technologique ou financière qui suppose des ressources politiques et financières peu banales, dont l'utilisation à des fins protectionnistes est tentante.

- La notion de développement durable. La notion se rapporte à la tentative, certes connue quant aux intentions, mais neuve dans la programmation régionale toscane, de donner à la contradiction potentielle entre développement et environnement une nouvelle perspective, susceptible de définir de nouvelles opportunités pour les acteurs publics et privés, dans le sens de l'innovation. Certes, dans le cas toscan, une telle énonciation d'objectifs est susceptible (et on l'a déjà constaté) de nourrir un débat fort long. Ici s'affrontent, d'un côté, la tradition industrielle de la région et, de l'autre, une sensibilité environnementale qui sort objectivement renforcée par le réalisme (réel ou supposé) de l'hypothèse postindustrielle. Celle-ci se fonde sur l'exceptionnalité évidente du patrimoine artistique et culturel régional auquel on entend confier le rôle moteur du développement. Mais cette notion de développement durable requiert, au moins autant que les autres, une utilisation extrêmement cohérente et entière dans sa phase de réalisation.

- Le choix de la programmation par le bas. Il constitue la tentative la plus évidente pour faire correspondre à l'innovation sociale et économique un changement cohérent du fonctionnement de l'innovation. Avec le PRS, qui anticipe ainsi l'imminence des nouvelles lois sur la programmation régionale, s'accomplit un pas vers une réforme sûrement pionnière dans le contexte italien.

Les choix du PRS s'articulent autour de deux instruments : le programme objectif (PO) et le programme d'initiative régionale (PIR). Le PO recense les ressources destinées à une mise en œuvre subsidiaire. On y accède par le bas à travers des programmes d'échelle subnationale. Les PIR concernent en revanche les actions qui exigent une intervention d'échelle régionale au moins. Ce sont typiquement des actions qui concernent les facteurs : innovation, crédit, promotion des exportations, etc.

Il s'agit évidemment d'une reproduction à l'échelle régionale du modèle européen : le PO fonctionne en analogie avec les fonds structurels, et les PIR avec les Plans d'Initiative Communautaire (PIC). La cohérence avec le scénario européen ne signifie pas que cette voie soit aisée. Depuis les premières expérimentations on a pu constater que le coût de l'inefficience devra être payé. Les institutions subrégionales, et notamment les provinces, sont appelées à exprimer une capacité de projet et de programmation 


\section{Pôle Sud $N^{\circ} 8$}

jusqu'ici jamais réellement exigée, sauf comme "strapontin" aux côtés d'entités supérieures.

Il revient à la région, en conséquence, de renforcer rapidement sa propre capacité de projet et celle des autres entités publiques. Beaucoup peut être fait en diffusant, avec la formation par exemple, une culture politico-administrative adaptée. À plus court terme, c'est du renforcement de l'outil technique dont il est question. Ainsi, auprès de l'IRPET, de l'agence financière (Fiditoscana) et de l'Agence pour les politiques d'innovation (qui devra consolider une mission remplie par le Réseau régional de la Haute Technologie ${ }^{9}$ ), on parle désormais de créer également une agence régionale pour le développement de "seconde génération", dans laquelle serait concentrée la "capacité de projet" de type "learning by doing". Cette transmission de culture de projet ne pourra se limiter aux entités publiques : elle doit nécessairement se diffuser dans la société pour susciter sur le territoire régional les conditions d'extension et d'enracinement de la méthode partenariale.

- Le rapport entre PRS et politiques européennes. Au cours des années, le PRS est parvenu à trouver dans les Documents Uniques de Programmation (DOCUP), prévus pour la mise en cuvre des fonds structurels européens (Objectifs 2 et $5 b$ ), un concurrent objectif dans la définition des lignes d'intervention régional, en raison surtout des dotations supérieures en ressources et du défaut de synchronie entre PRS et DOCUP. Le PRS 1998-2000 entend régler cette séparation entre les deux programmations, en définissant les priorités et critères de formulation des
DOCUP 2000-2006, non seulement pour faire apparaître la Toscane au rendez-vous de la réforme des fonds structurels, mais aussi pour clarifier les zones de dissension apparues dans certains aspects de programmes passés et formuler des propositions convaincantes à faire valoir dans la négociation région/Europe.

En particulier, la Toscane conteste la méthode de "zonisation", c'est-à-dire l'identification géographique des aires de soutien structurel. Cette méthode a d'abord eu des effets pervers (distorsion entre décision et allocation) induits par la différenciation des aides sur une base territoriale aux confins artificiels. En second lieu, une telle méthode suppose que les problèmes d'ajustement productifs de l'économie locale doivent trouver leur solution dans le contexte local lui-même. Ceci diminue d'autant la capacité de la région à donner aux problèmes locaux des réponses de niveau plus adapté, en manœuvrant en synergie avec toutes les opportunités offertes sur un territoire plus vaste.

En alternative à cette méthode, on propose une définition des systèmes locaux en fonction des bases structurelles de leur développement (aire industrielle de grande entreprise, PMI, zones touristiques, rurale, tertiaire) pour lesquelles sont articulées des interventions différentes.

- Le rapport entre PRS et budget. Le PRS 1998-2000 entend conclure le processus de convergence entre programmation et budget, en gouvernant directement une partie des ressources, mais sans renoncer à fournir les orientations stratégiques contraignantes sur l'ensemble de ces ressources. 


\section{La programmation du développement régional}

- La concertation. Elle est considérée comme une méthode ordinaire de processus politique, dépassant la pratique jusqu'alors prévalente des "consultations" des partenaires sociaux. Le PRS lui-même a été le résultat d'une ample réunion des acteurs institutionnels, économiques et sociaux. On y affirme, en d'autres termes, l'exigence de rechercher des choix communs sur des grands thèmes mais aussi celle d'une participation non-formelle, au sens où les ressources en jeu ne sont pas seulement régionales mais sont aussi celles détenues par les autres acteurs sociaux. Ainsi la concertation signifie aussi co-responsabilité dans la mise en œuvre. En Toscane d'ailleurs, des étapes significatives ont déjà été accomplies en ce sens, parmi lesquelles se distinguent les Pactes Territoriaux de Développement, labelisés en août 1996 par la région et tous ses partenaires, et que le PRS veut consolider en les plaçant au centre de la concertation.

Le PRS intervient à un moment charnière du parcours d'innovation institutionnelle et politique qui a plusieurs dimensions liées. La région expérimente en fait, notamment vis-à-vis des autres cas italiens, une logique d'anticipation dans l'application du principe de subsidiarité. Ces dernières années, elle s'est dépouillée de toutes les fonctions d'administration active, sauf celles liées aux PIR, en les attribuant aux gouvernements locaux. Cela a eu pour effet une diminution radicale des effectifs.
Même sur un terrain classique du pouvoir local comme la planification territoriale, elle s'en est remise à une nouvelle loi régionale d'urbanisme qui confie toutes les compétences à la province et aux communes et instaure une procédure accélérée pour l'approbation des plans d'urbanisme par la région. À l'intérieur de cette "machine" régionale, on a introduit une innovation révolutionnaire avec la séparation nette entre politique et administration qui réserve à la Junte régionale les actes de direction politique et de programmation mais en confie les pouvoirs de dépense aux dirigeants de la seconde.

Tout ceci, encore une fois, converge pour dessiner pour les prochaines années une nécessaire, mais non pour autant facile, phase d'expérimentations et d'apprentissages aux divers niveaux institutionnels et fonctionnels. Il s'agit d'une tentative qui n'a pas de précédent, au moins dans l'histoire des régions et des instances locales en Italie. Face à cette pression au changement demeure l'inconnue du régionalisme inachevé, jusqu'ici non dépassé, et l'attente, chargée d'incertitude sur les délais et le contenu, du fédéralisme promis qui devra découler du processus de réforme constitutionnelle mis en ouvre en Italie. Le pari de la Toscane est cependant que régionalisme et fédéralisme nécessitent en cette période de courageuses innovations "par le bas" avant et plus qu'une dévolution "octroyée" 10 par l'État national. 


\section{Pôle $S$ ud $N^{\circ} 8$}

\section{Notes}

1. Entre la fin des années 1950 et le début des années 1960 se créent les instituts régionaux de recherche, sans les régions, généralement initiées conjointement par les provinces, les communes et les chambres de commerce. Ce sont des instituts qui se sont souvent montrés de haut niveau : en voici la liste et les dates de fondation : Veneto (Irsev, 1957), Piemonte (Ires, 1958), Umbria (Crures, 1960), Lombardia (Ilses, 1960), Toscana (Itres, 1962 - Irpet depuis 1968), Liguria (Ilres, 1963), Marche (Issem, 1964).

2. Le travail de Becattini (1975) et de l'Irpet dont il était directeur représente la première étude absolue du courant d'analyse du développement local qui donnera lieu ensuite à des conceptualisations telles que la "Troisième Italie" (Bagnasco, 1977) et de l'Italie Nec (Nord-Est-Centro : Fuà, 1983). Pour une synthèse en anglais, $c f$. Becattini, 1978.

3. Le terme, destiné à avoir un succès retentissant dans la littérature italienne et internationale, est forgé dans Becattini, 1979. La conceptualisation a été remaniée en anglais dans Becattini, 1990.

4. Le gouvernement travailliste de Wilson lance la politique de programmation structurelle (structure planning) à l'échelle métropolitaine et régionale, inspirée par un manuel célébrissime sur la forme et le contenu des plans (DOE : Department of Environment, 1970).

5. Le système de modèles est principalement orienté vers la prévision à moyen terme, l'évaluation des politiques, la planification. Il peut en fait être utilisé pour la "production de prévisions économiques consistantes", avec référence à l'économie toscane. Quand il est utilisé comme "instrument d'évaluation des politiques", les prévisions deviennent conditionnelles en insérant dans le système des modèles des options sur les actions politiques. Si le système est employé comme "instrument de planification", on doit y introduire des paramètres pour la sélection des interventions politiques qui satisfassent à des critères de prestation, des choix de préférences ou des objectifs donnés.

6. En Toscane, dans la seconde législature (1975-1980), la programmation s'était déjà rapprochée de la Communauté Européenne dans le but d'y obtenir la légitimation des contenus du PRS. Cette opération ne réussit que partiellement, tant il est vrai que si les ressources européennes en question étaient déjà importantes, elles avaient un impact territorial limité (à l'agriculture et aux fonds sociaux) et relevaient avant tout d'une négociation nationale qui excluait les régions (Ferelli, 1981).

7. A la base de la nouvelle ère de programmation économique en Toscane se trouve une nouvelle série de travaux qui ont engendré, à partir de diverses prospectives, un schéma radicalement nouveau des connaissances sur la nature et les tendances des processus de développement économique et social. Ce sont les études récentes de l'IRPET pour une nouvelle interprétation du développement toscan; une recherche conjointe de l'IRPET et de la Scuola Superiore Sant'Anna de Pise sur l'industrie toscane (Varaldo, Bellini et Bonaccorsi, 1997) et des enquêtes réalisées sur les processus d'innovation (Regione Toscana, 1996)

8. La "maturité précoce" fait allusion au fait que l'économie de la Toscane qui était en 1955 encore essentiellement agricole n'est déjà plus essentiellement industrielle dès 1977. Le passage raide (20 ans) de l'état préindustriel au post-industriel a eu comme conséquence un niveau technologique faible dans l'industrie, un déclin rapide du taux d'industrialisation, un tertiaire carent dans ses parties les plus avancées (Bianchi, 1986).

9. Ce réseau (Regione Toscana, 1996) constitue une expérience intéressante de coordination (sans constitution d'une structure subordonnée) des trois pôles de recherche toscans (Florence, Pise et Sienne). Il opère depuis trois ans et a déjà mis en œuvre une trentaine de projets (Bianchi, 1996).

10. Ndt : en français dans le texte. 


\section{La programmation du développement régional}

\section{Références}

Becattini G. (dir.), Lo sviluppo economico della Toscana, Firenze, Irpet-Guaraldi, 1975.

Becattini G., "The Development of Light Industry in Tuscany : an Interpretation", Economic Notes, $\mathrm{n}^{\circ}$ 2-3, 1978.

Becattini G., " $\mathrm{Dal}$ 'settore industriale'al 'distretto industriale'. Alcune considerazioni sull'unità d'indagine dell'economia industriale", Rivista di economia e politica industriale, $\mathrm{n}^{\circ} 1,1979$.

Becattini G., "The Marshallian industrial district as a socio-economic notion", in Pyke F., Becattini G. et Sengenberger W. (eds.), Industrial Districts and Inter-Firm Cooperation in Italy, Genève, International Institute for Labour Study, 1990.

Bellini N. (ed.), "Industrial Districts", European Planning Studies, n 4, 1996.

Bianchi G., "L'esperienza di programmazione regionale in Italia : una breve rassegna critica", in Bielli M. et La Bella A. (dir.), Problematiche dei livelli sub-regionali di programmazione, Franco Angeli, Milano, 1982.

Bianchi G., "The Tuscany Case Study within Iiasa's Experience", in Iiasa-Irpet (ed.), Model Systems for Regional Planning-Regional Applied Systems Analysis : an International Appraisal, Luxembourg, liasa, 1983.

Bianchi G., "Maturità precoce : una modernizzazione a rischio", in Mori G. (dir.), Storia d'Italia. Le regioni dall'Unità a oggi. La Toscana, Einaudi, Torino, 1986.

Bianchi G., "Beautiful Music Badly Played : The Integrated Mediterranean Programmes : An Appraisal of Planning Design", Report prepared for DG XVI of the EC Commission within The European Community and the Regions Project, Florence, European University Institute, 1990.

Bianchi G., "The IMPSs : a Missed Opportunity? An Appraisal of the Design and Implementation of the Integrated Mediterranean Programmes", Regional Politics \& Policy, vol. 2, $\mathrm{n}^{\circ} 1-2,1992$.

Bianchi G., "Galileo used to live here. Tuscany hi-tech : the network and its poles", $R \& D$ Management, vol. 26, n³, 1996.

Carabba M., Vent'anni di programmazione : 1954-1974, Bari, Laterza, 1977.

Cavalieri A., Martellato D. Snickars F., Il sistema di modelli per lo studio di caso della Toscana : struttura dei singoli modelli e delle loro interrelazioni, in Leonardi G., Rabino G. A. (dir.), L'analisi degli insediamenti umani e produttivi, Milano, Franco Angeli, 1984.

DoE (Department of Environment), Development Plans. A Manual on Form and Content, London, Hmso, 1970.

Ferrelli N., La prospettiva istituzionale europea e $i$ riflessi finanziari della programmazione regionale, Firenze, Irpet-Le Monnier, 1981.

Fuà G., "L'industrializzazione nel Nord-Est e nel Centro", in Fuà G., Zacchia C. (dir.), Industrializzazione senza fratture, Bologna, Il Mulino, 1983.

Regione Toscana - Giunta regionale, Rete regionale dell'alta tecnologia. Progetto di fattibilità, Firenze, 1996.

Ruffolo G., "Sul piano Vanoni", in Carabba M. (dir.), Mezzogiorno e programmazione 1954/71, Milano, Giuffrè, 1980.

Saraceno P., "La mancata unificazione economica italiana a cento anni dall'unificazione politica", L'economia italiana dal 1861 al 1961, Milano, Giuffre, 1961.

Varaldo R., Bellini N., Bonaccorsi A., Tendenze e vie di cambiamento dell'industria toscana, Milano, Franco Angeli, 1997. 